\title{
A Note on the Ordinal Analysis of KPM
}

\author{
W. BUCHHOLZ
}

Mathematisches Institut der Universität München ${ }^{1}$

This note extends our method from (Buchholz [2]) in such a way that it applies also to the rather strong theory KPM. This theory was introduced and analyzed proof-theoretically in (Rathjen [6]), where Rathjen establishes an upper bound for its proof theoretic ordinal $|\mathrm{KPM}|$. The bound was given in terms of a primitive recursive system $\mathcal{T}(\mathrm{M})$ of ordinal notations based on certain ordinal functions $\chi, \psi_{\kappa}(\omega<\kappa<\mathrm{M}, \kappa \text { regular })^{2}$ that had been introduced and studied in (Rathjen [5]). ${ }^{3}$ In section 1 of this note we define and study a slightly different system of functions $\psi_{\kappa}(\kappa \leq \mathrm{M})$ - where $\psi_{\mathrm{M}}$ plays the rôle of Rathjen's $\chi$ - that is particularly well suited for our purpose of extending [2]. In section 2 we describe how one obtains, by a suitable modification of [2], an upper bound for $|\mathrm{KPM}|$ in terms of the $\psi_{\kappa}$ 's from section 1. We conjecture that this bound is best possible and coincides with the bound given in [6]. In section 3 we prove some additional properties of the functions $\psi_{\kappa}$ which are needed to set up a primitive recursive ordinal notation system of ordertype $>\vartheta^{\star}$, where $\vartheta^{\star}:=\psi_{\Omega_{1}} \varepsilon_{\mathrm{M}+1}$ is the upper bound for $|\mathrm{KPM}|$ determined in section 2.

\section{Remark}

Another ordinal analysis of KPM has been obtained independently by T. Arai in "Proof theory for reflecting ordinals II: recursively Mahlo ordinals." (Handwritten notes, 1989).

\footnotetext{
${ }^{1}$ The final version of this paper was written while the author was visiting Carnegie Mellon University during the academic year 1990/91. I would like to express my sincere thanks to Wilfried Sieg (who invited me) and all members of the Philosophy Department of $\mathrm{CMU}$ for their generous hospitality.

${ }^{2} \mathrm{M}$ denotes the first weakly Mahlo cardinal.

${ }^{3}$ The essential new feature of [5] is the function $\chi$, while the $\psi_{\kappa}$ 's $(\kappa<\mathrm{M})$ are obtained by a straightforward generalization of previous constructions in [1],[3],[4].
} 


\section{Basic properties of the functions $\psi_{\kappa}(\kappa \leq \mathrm{M})$}

\section{Preliminaries}

The letters $\alpha, \beta, \gamma, \delta, \mu, \sigma, \xi, \eta, \zeta$ always denote ordinals. On denotes the class of all ordinals, and Lim the class of all limit numbers. Every ordinal $\alpha$ is identified with the set $\{\xi \in$ On $: \xi<\alpha\}$ of its predecessors. For $\alpha \leq \beta$ we set $[\alpha, \beta[:=\{\xi: \alpha \leq \xi<\beta\}$. By + we denote ordinary (noncommutative) ordinal addition. An ordinal $\alpha>0$ which is closed under + is called an additive principal number. The class of all additive principal numbers is denoted by AP. The Veblen function $\varphi$ is defined by $\varphi \alpha \beta:=\varphi_{\alpha}(\beta)$, where $\varphi_{\alpha}$ is the ordering function of the class $\left\{\beta \in \mathrm{AP}: \forall \xi<\alpha\left(\varphi_{\xi}(\beta)=\beta\right)\right\}$. An ordinal $\gamma>0$ which is closed under $\varphi$ (and thus also under + ) is said to be strongly critical. The class of all strongly critical ordinals is denoted by SC.

Some basic facts:

1. $\mathrm{AP}=\left\{\omega^{\alpha}: \alpha \in \mathrm{On}\right\}$

2. $\varphi 0 \beta=\omega^{\beta}, \varphi 1 \beta=\varepsilon_{\beta}$

3. For each $\gamma>0$ there are uniquely determined $n \in \mathbb{N}$ and additive principal numbers $\gamma_{0} \geq \ldots \geq \gamma_{n}$ such that $\gamma=\gamma_{0}+\ldots+\gamma_{n}$.

4. For each $\gamma \in \mathrm{AP} \backslash \mathrm{SC}$ there are uniquely determined $\xi, \eta<\gamma$ such that $\gamma=\varphi \xi \eta$.

5. Every uncountable cardinal is strongly critical.

Definition of $S C(\gamma)$

1. $S C(0):=\emptyset$

2. $S C(\gamma):=\{\gamma\}$, if $\gamma \in \mathrm{SC}$

3. $S C\left(\gamma_{0}+\ldots+\gamma_{n}\right):=S C\left(\gamma_{0}\right) \cup \ldots \cup S C\left(\gamma_{n}\right)$, if $n \geq 1$ and $\gamma_{0} \geq \ldots \geq \gamma_{n}$ are additive principal numbers.

4. $S C(\varphi \xi \eta):=S C(\xi) \cup S C(\eta)$, if $\xi, \eta<\varphi \xi \eta$.

We assume the existence of a weakly Mahlo cardinal M.

So every closed unbounded (club) set $X \subseteq \mathrm{M}$ contains at least one regular cardinal, and $\mathrm{M}$ itself is a regular cardinal. 


\section{Definition 1.1}

$\mathrm{R}:=\{\alpha: \omega<\alpha \leq \mathrm{M} \& \alpha$ regular $\}$

$\mathrm{M}^{\Gamma}:=\min \{\gamma \in \mathrm{SC}: \mathrm{M}<\gamma\}=$ closure of $\mathrm{M} \cup\{\mathrm{M}\}$ under,$+ \varphi$

$S C_{\mathrm{M}}(\gamma):=S C(\gamma) \cap \mathrm{M}$

$\Omega_{0}:=0, \Omega_{\sigma}:=\aleph_{\sigma}$ for $\sigma>0$.

$\boldsymbol{\Omega}:=$ the function $\sigma \mapsto \Omega_{\sigma}$ restricted to $\sigma<\mathrm{M}$

Remark: $\forall \kappa \in \mathrm{R}\left(\kappa=\Omega_{\kappa}\right.$ or $\left.\kappa \in\left\{\Omega_{\sigma+1}: \sigma<\mathrm{M}\right\}\right)$

Convention. In the following the letters $\kappa, \pi, \tau$ always denote elements of $\mathrm{R}$.

Definition 1.2 (The collapsing functions $\psi_{\kappa}$ )

By transfinite recursion on $\alpha$ we define ordinals $\psi_{\kappa} \alpha$ and sets $C(\alpha, \beta) \subseteq$ On as follows. Under the induction hypothesis that $\psi_{\pi} \xi$ and $C(\xi, \eta)$ are already defined for all $\xi<\alpha, \pi \in \mathrm{R}, \eta \in \mathrm{O}$ we set

1. $C(\alpha, \beta):=$ closure of $\beta \cup\{0, \mathrm{M}\}$ under $+, \varphi, \Omega, \psi \mid \alpha$,

where $\psi \mid \alpha$ denotes the binary function given by

$$
\begin{aligned}
& \operatorname{dom}(\psi \mid \alpha):=\left\{(\pi, \xi): \xi<\alpha \& \pi \in \mathrm{R} \& \pi, \xi \in C\left(\xi, \psi_{\pi} \xi\right)\right\} \\
& (\psi \mid \alpha)(\pi, \xi):=\psi_{\pi} \xi .
\end{aligned}
$$

2. $\psi_{\kappa} \alpha:=\min \left\{\beta \in \mathcal{D}_{\kappa}(\alpha): C(\alpha, \beta) \cap \kappa \subseteq \beta\right\}$

$$
\text { with } \quad \mathcal{D}_{\kappa}(\alpha):= \begin{cases}\{\beta \in \mathrm{R}: \alpha \in C(\alpha, \mathrm{M}) \Rightarrow \alpha \in C(\alpha, \beta)\} & \text { if } \kappa=\mathrm{M} \\ \{\beta: \kappa \in C(\alpha, \kappa) \Rightarrow \kappa \in C(\alpha, \beta)\} & \text { if } \kappa<\mathrm{M}\end{cases}
$$

Abbreviation: $C_{\kappa}(\alpha):=C\left(\alpha, \psi_{\kappa} \alpha\right)$

The first two lemmata are immediate consequences of Definition 1.2.

\section{Lemma 1.1}

a) $\alpha_{0} \leq \alpha \& \beta_{0} \leq \beta \Longrightarrow C\left(\alpha_{0}, \beta_{0}\right) \subseteq C(\alpha, \beta)$

b) $\emptyset \neq X \subseteq$ On \& $\beta=\sup (X) \Longrightarrow C(\alpha, \beta)=\bigcup_{\eta \in X} C(\alpha, \eta)$

c) $\beta<\kappa \Longrightarrow \operatorname{card}(C(\alpha, \beta))<\kappa$

\section{Lemma 1.2}

$C(\alpha, \beta)=\bigcup_{n<\omega} C^{n}(\alpha, \beta)$, where $C^{n}(\alpha, \beta)$ is defined by

(i) $C^{0}(\alpha, \beta):=\beta \cup\{0, \mathrm{M}\}$,

(ii) $C^{n+1}(\alpha, \beta):=\left\{\gamma: S C(\gamma) \subseteq C^{n}(\alpha, \beta)\right\} \cup\left\{\Omega_{\sigma}: \sigma \in C^{n}(\alpha, \beta)\right\} \cup$

$$
\cup\left\{\psi_{\pi} \xi: \xi<\alpha \& \pi, \xi \in C^{n}(\alpha, \beta) \cap C_{\pi}(\xi)\right\}
$$




\section{Lemma 1.3}
a) $C_{\kappa}(\alpha) \cap \kappa=\psi_{\kappa} \alpha<\kappa$
b) $\kappa<\mathrm{M} \Longrightarrow \psi_{\kappa} \alpha \notin \mathrm{R}$
c) $\psi_{\kappa} \alpha \in \mathrm{SC} \backslash\left\{\Omega_{\sigma}: \sigma<\Omega_{\sigma}\right\}$
d) $\kappa \in C(\alpha, \kappa) \Longleftrightarrow \kappa \in C_{\kappa}(\alpha)$
e) $C(\alpha, \mathrm{M})=\mathrm{M}^{\Gamma}=\left\{\xi: \xi \in C_{\mathrm{M}}(\xi)\right\}$
f) $\gamma \in C_{\kappa}(\alpha) \Longrightarrow \gamma \in C_{\mathrm{M}}(\gamma) \& S C_{\mathrm{M}}(\gamma)=S C(\gamma) \backslash\{\mathrm{M}\}$
g) $\gamma<\alpha \& \gamma \in C(\alpha, \beta) \Longrightarrow \psi_{\mathrm{M}} \gamma \in C(\alpha, \beta)$

Proof.

a),b) 1. $C_{\kappa}(\alpha) \cap \kappa=\psi_{\kappa} \alpha$ is a trivial consequence of the definition of $\psi_{\kappa} \alpha$.

2. Let $\kappa=$ M. Obviously there exists a $\delta<\kappa$ such that $\mathrm{R} \cap[\delta, \kappa[\subseteq$ $\mathcal{D}_{\kappa}(\alpha)$. Therefore in order to get $\psi_{\kappa} \alpha<\kappa$ it suffices to prove that the set $U:=\{\beta \in \kappa: C(\alpha, \beta) \cap \kappa \subseteq \beta\}$ is closed unbounded (club) in $\kappa$.

i) closed: Let $\emptyset \neq X \subseteq U$ and $\beta:=\sup (X)<\kappa$. Then $C(\alpha, \beta) \cap \kappa=$ $\bigcup_{\xi \in X}(C(\alpha, \xi) \cap \kappa) \subseteq \bigcup_{\xi \in X} \xi=\beta$, i.e. $\beta \in U$.

ii) unbounded: Let $\beta_{0}<\kappa$. We define $\beta_{n+1}:=\min \left\{\eta: C\left(\alpha, \beta_{n}\right) \cap \kappa \subseteq \eta\right\}$ and $\beta:=\sup _{n<\omega} \beta_{n}$. Using L.1.1c we obtain $\beta_{n} \leq \beta_{n+1}<\kappa$. Hence $\beta_{0} \leq \beta<\kappa$ and $C(\alpha, \beta) \cap \kappa=\bigcup_{n<\omega}\left(C\left(\alpha, \beta_{n}\right) \cap \kappa\right) \subseteq \bigcup_{n<\omega} \beta_{n+1}=\beta$, i.e. $\beta_{0} \leq \beta \in U$.

3. Let $\kappa<\mathrm{M}$. Starting with $\beta_{0}:=\min \left(\mathcal{D}_{\kappa}(\alpha)\right)$ we define the ordinals $\beta_{n}$ and $\beta$ as in 2.(ii). Then we have $\beta \in \mathcal{D}_{\kappa}(\alpha) \cap U$ and therefore $\psi_{\kappa} \alpha \leq \beta<\kappa$. - Now assume that $\psi_{\kappa} \alpha \in \mathrm{R}$. We prove $\beta_{n}<\psi_{\kappa} \alpha(\forall n)$. By definition of $\beta_{0}$ and by L.1.1a we have $\beta_{0} \leq \psi_{\kappa} \alpha \& \beta_{0} \notin \operatorname{Lim}$. Hence $\beta_{0}<\psi_{\kappa} \alpha$. From $\beta_{n}<\psi_{\kappa} \alpha \in \mathrm{R}$ it follows that $C\left(\alpha, \beta_{n}\right) \cap \kappa \subseteq \psi_{\kappa} \alpha$ and $\operatorname{card}\left(C\left(\alpha, \beta_{n}\right) \cap \kappa\right)<\psi_{\kappa} \alpha$, and therefore $\beta_{n+1}<\psi_{\kappa} \alpha$. From $\forall n\left(\beta_{n}<\psi_{\kappa} \alpha \in \mathrm{R}\right)$ we get $\beta<\psi_{\kappa} \alpha$. Contradiction.

c) 1. Obviously $C_{\kappa}(\alpha) \cap \kappa$ is closed under $\varphi$. Together with a) this implies $\psi_{\kappa} \alpha \in$ SC. - 2. We have $\left(\psi_{\kappa} \alpha=\Omega_{\sigma}>\sigma \Rightarrow \psi_{\kappa} \alpha \in C_{\kappa}(\alpha)\right)$ and (by a) ) $\psi_{\kappa} \alpha \notin C_{\kappa}(\alpha)$. Hence $\psi_{\kappa} \alpha \notin\left\{\Omega_{\sigma}: \sigma<\Omega_{\sigma}\right\}$.

d) follows from L.1.1a, L.1.3a and the definition of $\psi_{\kappa} \alpha$.

e) By L.1.3a $\forall \pi \in \mathrm{R}\left(\psi_{\pi} \xi<\mathrm{M}\right)$ and therefore $C(\alpha, \mathrm{M})=\mathrm{M}^{\Gamma}$. As in d) one obtains $\left(\alpha \in C(\alpha, \mathrm{M}) \Leftrightarrow \alpha \in C_{\mathrm{M}}(\alpha)\right)$.

f) and g) follow from e). 


\section{Lemma 1.4}
a) $\gamma \in C(\alpha, \beta) \Longleftrightarrow S C(\gamma) \subseteq C(\alpha, \beta)$
b) $\Omega_{\sigma} \in C(\alpha, \beta) \Longleftrightarrow \sigma \in C(\alpha, \beta)$
c) $\kappa=\Omega_{\sigma+1} \Longrightarrow \Omega_{\sigma}<\psi_{\kappa} \alpha<\Omega_{\sigma+1}$
d) $\Omega_{\kappa}=\kappa \Longrightarrow \Omega_{\psi_{\kappa} \alpha}=\psi_{\kappa} \alpha$
e) $\Omega_{\psi_{\mathrm{M}} \alpha}=\psi_{\mathrm{M}} \alpha$
f) $\Omega_{\sigma} \leq \gamma \leq \Omega_{\sigma+1} \& \gamma \in C(\alpha, \beta) \Longrightarrow \sigma \in C(\alpha, \beta)$

Proof. a) and b) follow from L.1.2 and L.1.3c. - e) follows from d), since $\mathrm{M} \in \mathrm{R}$ and $\Omega_{\mathrm{M}}=\mathrm{M}$. - f) follows from a),b),c),d) and L.1.2.

c) Let $\kappa=\Omega_{\sigma+1}$. Then $\kappa \in C(\alpha, \kappa)$ and thus $\kappa \in C_{\kappa}(\alpha)$. By a) and b) from $\kappa=\Omega_{\sigma+1} \in C_{\kappa}(\alpha)$ we get $\Omega_{\sigma} \in C_{\kappa}(\alpha) \cap \kappa=\psi_{\kappa} \alpha$.

d) Take $\sigma \in$ On such that $\Omega_{\sigma} \leq \psi_{\kappa} \alpha<\Omega_{\sigma+1}$. Then we have $\sigma+1<\kappa$ and thus $C_{\kappa}(\alpha) \cap \kappa=\psi_{\kappa} \alpha<\Omega_{\sigma+1}<\Omega_{\kappa}=\kappa$. This implies $\Omega_{\sigma+1} \notin C_{\kappa}(\alpha)$ and then (by a),b) ) $\sigma \notin C_{\kappa}(\alpha)$. Hence $\psi_{\kappa} \alpha \leq \sigma \leq \Omega_{\sigma} \leq \psi_{\kappa} \alpha$.

\section{Lemma 1.5}

a) $\alpha_{0}<\alpha \& \alpha_{0} \in C_{\mathrm{M}}(\alpha) \Longrightarrow \psi_{\mathrm{M}} \alpha_{0}<\psi_{\mathrm{M}} \alpha$

b) $\psi_{\mathrm{M}} \alpha_{0}=\psi_{\mathrm{M}} \alpha_{1} \& \alpha_{0}, \alpha_{1}<\mathrm{M}^{\Gamma} \Longrightarrow \alpha_{0}=\alpha_{1}$

Proof.

a) From the premise we get $\psi_{\mathrm{M}} \alpha_{0} \in C_{\mathrm{M}}(\alpha) \cap \mathrm{M}=\psi_{\mathrm{M}} \alpha$ by L.1.3a,g.

b) Assume $\psi_{\mathrm{M}} \alpha_{0}=\psi_{\mathrm{M}} \alpha_{1} \& \alpha_{0}<\alpha_{1}<\mathrm{M}^{\Gamma}$. Then $\alpha_{0} \in C_{\mathrm{M}}\left(\alpha_{0}\right) \subseteq C_{\mathrm{M}}\left(\alpha_{1}\right)$ and therefore by a) $\psi_{\mathrm{M}} \alpha_{0}<\psi_{\mathrm{M}} \alpha_{1}$. Contradiction.

\section{Lemma 1.6}

For $\kappa<\mathrm{M}$ the following holds

a) $\alpha_{0}<\alpha \Longrightarrow \psi_{\kappa} \alpha_{0} \leq \psi_{\kappa} \alpha$

b) $\alpha_{0}<\alpha \& \kappa, \alpha_{0} \in C_{\kappa}\left(\alpha_{0}\right) \Longrightarrow \psi_{\kappa} \alpha_{0}<\psi_{\kappa} \alpha$

Proof.

a) From $\alpha_{0}<\alpha$ it follows that $C\left(\alpha_{0}, \psi_{\kappa} \alpha\right) \cap \kappa \subseteq \psi_{\kappa} \alpha$. By definition of $\psi_{\kappa} \alpha_{0}$ it therefore suffices to prove $\psi_{\kappa} \alpha \in\left\{\beta: \kappa \in C\left(\alpha_{0}, \kappa\right) \Rightarrow \kappa \in C\left(\alpha_{0}, \beta\right)\right\}$. So let $\kappa \in C\left(\alpha_{0}, \kappa\right)$. - We have to prove $\kappa \in C\left(\alpha_{0}, \psi_{\kappa} \alpha\right)$.

CASE 1: $\kappa=\Omega_{\sigma+1}$. By Lemma 1.4c we have $\Omega_{\sigma}<\psi_{\kappa} \alpha$ and therefore $\sigma+1 \in C\left(\alpha_{0}, \psi_{\kappa} \alpha\right)$ which implies $\kappa \in C\left(\alpha_{0}, \psi_{\kappa} \alpha\right)$. 
CASE 2: $\kappa=\Omega_{\kappa}$. From $\kappa \in C\left(\alpha_{0}, \kappa\right) \subseteq C(\alpha, \kappa)$ we obtain $\kappa \in C_{\kappa}\left(\alpha_{0}\right) \cap C_{\kappa}(\alpha)$. From this by L.1.2, L.1.3b, L.1.5b it follows that $\kappa=\psi_{\mathrm{M}} \xi$ with $\xi<\alpha_{0}$ and $\xi \in C_{\kappa}(\alpha)$. Now by L.1.4a, L.1.3a,e we get $S C_{\mathrm{M}}(\xi) \subseteq C_{\kappa}(\alpha) \cap C_{\mathrm{M}}(\xi) \cap \mathrm{M}=$ $C_{\kappa}(\alpha) \cap \kappa=\psi_{\kappa} \alpha$, and then $\xi \in C\left(\alpha_{0}, \psi_{\kappa} \alpha\right)$ (by L.1.3f). From this together with $\xi<\alpha_{0}$ we obtain $\kappa=\psi_{\mathrm{M}} \xi \in C\left(\alpha_{0}, \psi_{\kappa} \alpha\right)$ (by L.1.3g).

b) The premise together with a) implies $\alpha_{0}<\alpha \& \kappa, \alpha_{0} \in C_{\kappa}(\alpha) \cap C_{\kappa}\left(\alpha_{0}\right)$ which gives us $\psi_{\kappa} \alpha_{0} \in C_{\kappa}(\alpha) \cap \kappa=\psi_{\kappa} \alpha$.

\section{Definition 1.3}

For each set $X \subseteq$ On we set $\mathcal{H}_{\gamma}(X):=\bigcap\{C(\alpha, \beta): X \subseteq C(\alpha, \beta) \& \gamma<\alpha\}$.

\section{Ordinal analysis of KPM}

In this section we show how one has to modify (and extend) [2] in order to establish that the ordinal $\psi_{\Omega_{1}} \varepsilon_{\mathrm{M}+1}$ is an upper bound for $|\mathrm{KPM}|$. Of course we now assume that the reader is familiar with [2].

The theory KPM is obtained from KPi by adding the following axiom scheme: (Mahlo) $\forall x \exists y \phi(x, y, \vec{z}) \rightarrow \exists w[A d(w) \wedge \forall x \in w \exists y \in w \phi(x, y, \vec{z})] \quad\left(\phi \in \Delta_{0}\right)$

We extend the infinitary system $\mathrm{RS}^{\infty}$ introduced in Section 3 of [2] by adding the following inference rule:

(Mah) $\frac{\Gamma, B\left(\mathrm{~L}_{\mathrm{M}}\right): \alpha_{0}}{\Gamma, \exists w \in \mathrm{L}_{\mathrm{M}}(A d(w) \wedge B(w)): \alpha} \quad\left(\alpha_{0}+\mathrm{M}<\alpha\right)$

where $B(w)$ is of the form $\forall x \in w \exists y \in w A(x, y)$ with $\mathrm{k}(A) \subseteq \mathrm{M}$.

We set $\mathrm{R}:=\{\alpha: \omega<\alpha \leq \mathrm{M} \& \alpha$ regular $\}$.

Then all lemmata and theorems of Section $3^{4}$ are also true for the extended system $\mathrm{RS}^{\infty}$ (with almost literally the same proofs) ${ }^{5}$, and as an easy consequence from Theorem $\mathbf{3 . 1 2}$ one obtains the

\footnotetext{
${ }^{4}$ We use boldface numerals to indicate reference to [2]

${ }^{5}$ In Theorem 3.8 one has to add the clause which corresponds to the new inference rule (Mah). The last line in the proof of Lemma $\mathbf{3 . 1 4}$ has to be modified to "... cannot be the main part of a (Ref)- or (Mah)-inference.". At the end of the proof of Lemma $\mathbf{3 . 1 7}$ one may add the remark "Due to the premise $\alpha \leq \beta<\kappa$ we have $\alpha<\mathrm{M}$, and therefore the given derivation of $\Gamma, C$ does not contain any applications of (Mah).".
} 
Embedding Theorem for KPM

If $\mathrm{M} \in \mathcal{H}$ and if $\mathcal{H}$ is closed under $\xi \mapsto \xi^{\mathrm{R}}$ then for each theorem $\phi$ of $\mathrm{KPM}$ there is an $n \in \mathbb{N}$ such that $\mathcal{H} \mid \frac{\omega^{\mathrm{M}+n}}{\mathrm{M}+n} \phi^{\mathrm{M}}$.

Some more severe modifications have to be carried out on Section 4 . The first part of this section (down to Lemma 4.5) has to be replaced by Section 1 of the present paper. Then the sets $C(\alpha, \beta)$ are no longer closed under $(\pi, \xi) \mapsto \psi_{\pi} \xi \quad(\xi<\alpha)$, but only under $(\psi \mid \alpha)$ as defined in Definition 1.2 above. Therefore we have to add " $\pi, \xi \in C_{\pi}(\xi)$ " to the premise of Lemma 4.6c, and accordingly a minor modification as to be made in the proof of Lemma 4.7 $(\mathcal{A} 1)$. But this causes no problems. A little bit problematic is the fact that the function $\psi_{\mathrm{M}}$ is not weakly increasing. In order to overcome this difficulty we prove the following lemma.

\section{Definition 2.1}

For $\gamma=\omega^{\gamma_{0}}+\ldots+\omega^{\gamma_{n}}$ with $\gamma_{0} \geq \ldots \geq \gamma_{n}$ we set $\mathrm{e}(\gamma):=\omega^{\gamma_{n}+1}$.

Further we set $\mathrm{e}(0):=$ On.

\section{Lemma 2.1}

For $\gamma \in C_{\mathrm{M}}(\gamma+1)$ and $0<\alpha<\mathrm{e}(\gamma)$ the following holds

a) $\psi_{\mathrm{M}}(\gamma+1) \leq \psi_{\mathrm{M}}(\gamma+\alpha) \& C_{\mathrm{M}}(\gamma+1) \subseteq C_{\mathrm{M}}(\gamma+\alpha)$

b) $0<\alpha_{0}<\alpha \& \alpha_{0} \in C_{\mathrm{M}}(\gamma+1) \Longrightarrow \psi_{\mathrm{M}}\left(\gamma+\alpha_{0}\right)<\psi_{\mathrm{M}}(\gamma+\alpha)$

Proof:

a) follows from b).

b) We will prove $(*) \psi_{\mathrm{M}}(\gamma+1) \leq \psi_{\mathrm{M}}(\gamma+\alpha)$. From this we get $\gamma+\alpha_{0} \in$ $C_{\mathrm{M}}(\gamma+1) \subseteq C_{\mathrm{M}}(\gamma+\alpha)$ and then by L.1.5a the assertion.

For $\gamma=0(*)$ is trivial. If $\gamma \neq 0$ then $\gamma+\alpha<\mathrm{M}^{\Gamma}$ and therefore $\gamma+\alpha \in$ $C_{\mathrm{M}}(\gamma+\alpha)$ which (together with $\left.\alpha<\mathrm{e}(\gamma)\right)$ implies $\gamma+1 \in C_{\mathrm{M}}(\gamma+\alpha)$. Hence $\psi_{\mathrm{M}}(\gamma+1) \leq \psi_{\mathrm{M}}(\gamma+\alpha)$ by L.1.5a.

Now we give a complete list of all modifications which have to be carried out in [2] subsequent to Lemma $\mathbf{4 . 6}$.

(1) Replace I by $\mathrm{M}$ in the definition of $\overline{\mathrm{K}}$.

(2) Add " $\eta<\gamma+\mathrm{e}(\gamma)$ " to the premise of Lemma 4.7( $\mathcal{A} 2)$.

(3) Add " $\omega^{\mu+\alpha}<\mathrm{e}(\gamma)$ " to the premise of Theorem 4.8.

(4) Add " $\pi \leq \mathrm{e}\left(\gamma^{\prime}\right)$ " to the premise of $(\square)$ in the proof of Theorem 4.8 . 
(5) Insert the following proof of " $\psi_{\kappa} \alpha^{*} \leq \psi_{\kappa} \widehat{\alpha}$ " at the end of the proof of ( $\square$ ): "From $\gamma^{\prime}, \mu^{\prime}, \alpha^{\prime} \in \mathcal{H}_{\gamma^{\prime}}[\Theta]$ we get $\alpha^{*} \in \mathcal{H}_{\gamma^{\prime}}[\Theta]$. From $\mathrm{k}(\Theta) \subseteq C_{\kappa}(\gamma+1) \subseteq$ $C_{\kappa}(\widehat{\alpha}) \& \gamma^{\prime}<\widehat{\alpha}$ it follows that $\mathcal{H}_{\gamma^{\prime}}[\Theta] \subseteq C_{\kappa}(\widehat{\alpha})$. Hence $\alpha^{*} \in C_{\kappa}(\widehat{\alpha})$ and thus $\psi_{\kappa} \alpha^{*} \leq \psi_{\kappa} \widehat{\alpha}$, since $\alpha^{*}<\widehat{\alpha}$."

(6) Extend the proof of Theorem $\mathbf{4 . 8}$ by the following treatment of the case where the last inference in the given derivation of $\Gamma$ is an application of (Mah):

"5. Suppose that $\exists w \in \mathrm{L}_{\mathrm{M}}(A d(w) \wedge B(w)) \in \Gamma$ and $\mathcal{H}_{\gamma}[\Theta] \mid \frac{\alpha_{0}}{\mu} \Gamma, B\left(\mathrm{~L}_{\mathrm{M}}\right)$ with $B(w) \equiv \forall x \in w \exists y \in w A(x, y) \quad \& \quad \alpha_{0}+\mathrm{M}<\alpha \quad \& \mathrm{k}(A) \subseteq \mathrm{M}$.

Then $\kappa=\mathrm{M}($ since $\Gamma \subseteq \Sigma(\kappa)$ and $\kappa \leq \mathrm{M})$.

For $\iota \in \mathcal{T}_{\mathrm{M}}$ we set $\gamma_{\iota}:=\gamma+\omega^{\mu+\alpha_{0}+|\iota|}$. Then $C_{\mathrm{M}}(\gamma+1) \subseteq C_{\mathrm{M}}\left(\gamma_{\iota}\right)$, and since $S C(|\iota|) \subseteq S C_{\mathrm{M}}\left(\gamma_{\iota}\right) \subseteq \psi_{\mathrm{M}} \gamma_{\iota}$, we have $|\iota|<\psi_{\mathrm{M}} \gamma_{\iota}$ and thus $\mathrm{k}(\Theta, \iota) \subseteq C_{\mathrm{M}}\left(\gamma_{\iota}\right)$. From $\gamma, \mu, \alpha_{0} \in \mathcal{H}_{\gamma}[\Theta]$ we get $\gamma_{\iota} \in \mathcal{H}_{\gamma}[\Theta, \iota]$. Consequently $\mathcal{A}\left(\Theta, \iota ; \gamma_{\iota}, \mathrm{M}, \mu\right)$, and the Inversion-Lemma gives us $\mathcal{H}_{\gamma}[\Theta][\iota] \mid \frac{\alpha_{0}}{\mu} \Gamma, \iota \notin \mathrm{L}_{0} \rightarrow \exists y \in \mathrm{L}_{\mathrm{M}} A(\iota, y)$.

Now we apply the I.H. and obtain $\mathcal{H}_{\alpha_{\iota}^{*}}[\Theta][\iota] \mid \frac{\psi_{\mathrm{M}} \alpha_{\iota}^{*}}{\cdot} \Gamma, \iota \notin \mathrm{L}_{0} \rightarrow \exists y \in \mathrm{L}_{\mathrm{M}} A(\iota, y)$ with $\alpha_{\iota}^{*}:=\gamma_{\iota}+\omega^{\mu+\alpha_{0}}<\gamma+\omega^{\mu+\alpha_{0}+\mathrm{M}}=: \alpha^{*}<\widehat{\alpha}$.

Let $\pi:=\psi_{\mathrm{M}} \alpha^{*} \& \beta_{\iota}:=\psi_{\mathrm{M}} \alpha_{\iota}^{*} . \quad$ Then by L.4.7 $\pi \in \mathcal{H}_{\widehat{\alpha}}[\Theta] \& \pi<\psi_{\mathrm{M}} \widehat{\alpha}$. We also have $\forall \iota \in \mathcal{T}_{\pi}\left(\alpha_{\iota}^{*} \in C_{\mathrm{M}}\left(\alpha^{*}\right)\right)$ and thus $\forall \iota \in \mathcal{T}_{\pi}\left(\beta_{\iota}<\pi\right)$.

The Boundedness-Lemma gives us now $\forall \iota \in \mathcal{T}_{\pi}\left(\mathcal{H}_{\hat{\alpha}}[\Theta][\iota] \mid \frac{\beta_{\iota}}{\pi} \Gamma, \iota \notin \mathrm{L}_{0} \rightarrow \exists y \in \mathrm{L}_{\pi} A(\iota, y)\right)$.

From this by an application of $(\Lambda)$ we obtain $\mathcal{H}_{\widehat{\alpha}}[\Theta] \mid \frac{\pi}{.} \Gamma, B\left(\mathrm{~L}_{\pi}\right)$.

From L.2.5h and L.3.10 we get $\mathcal{H}_{\widehat{\alpha}}[\Theta] \mid \frac{\delta}{0} \Gamma, A d\left(\mathrm{~L}_{\pi}\right)$ with $\delta:=\omega^{\pi+5}$. We also have $\mathcal{H}_{\widehat{\alpha}}[\Theta] \mid \frac{0}{.} \Gamma, \mathrm{L}_{\pi} \notin \mathrm{L}_{0}$. Hence $\mathcal{H}_{\widehat{\alpha}}[\Theta] \mid \frac{\delta+2}{\cdot} \Gamma, \mathrm{L}_{\pi} \notin \mathrm{L}_{0} \wedge A d\left(\mathrm{~L}_{\pi}\right) \wedge B\left(\mathrm{~L}_{\pi}\right)$. Now we apply $(\mathrm{V})$ and obtain $\mathcal{H}_{\widehat{\alpha}}[\Theta] \mid \frac{\psi_{\mathrm{M}} \widehat{\alpha}}{\cdot} \Gamma$."

(7) Replace I by M in the Corollary to Theorem 4.8 and in Theorem 4.9. This yields the following Theorem.

THEOREM

Let $\vartheta^{\star}:=\psi_{\Omega_{1}}\left(\varepsilon_{\mathrm{M}+1}\right)$. Then for each $\Sigma_{1}$-sentence $\phi$ of $\mathcal{L}$ we have:

$\mathrm{KPM} \vdash \forall x\left(\operatorname{Ad}(x) \rightarrow \phi^{x}\right) \quad \Longrightarrow \quad L_{\vartheta^{\star}} \models \phi$.

COROLLARY. $|\mathrm{KPM}| \leq \psi_{\Omega_{1}}\left(\varepsilon_{\mathrm{M}+1}\right)$. 


\section{Further properties of the functions $\psi_{\kappa}$}

We prove four theorems which together with L.1.3a,b,c and L.1.4a-e provide a complete basis for the definition of a primitive recursive wellordering $(\mathrm{OT}, \prec)$ which is isomorphic to $\left(C\left(\mathrm{M}^{\Gamma}, 0\right),<\right)$. (The set OT consists of terms built up from the constants $\underline{0}, \underline{\mathrm{M}}$ by the function symbols $\underline{ \pm}, \underline{\varphi}, \underline{\boldsymbol{\Omega}}, \underline{\psi}$, such that for each $\gamma \in C\left(\mathrm{M}^{\Gamma}, 0\right)$ there is a unique term $t \in \mathrm{OT}$ with $|t|=\gamma$, and for all $s, t \in \mathrm{OT}$ one has $(s \prec t \Leftrightarrow|s|<|t|)$. Here $|t|$ denotes the canonical value of $t$. For details see [1], [4], [5].)

Now the letters $\alpha, \beta, \gamma, \delta, \mu, \sigma, \xi, \eta, \zeta$ always denote ordinals less than $\mathrm{M}^{\Gamma}$. So, for all $\alpha$ we have $\alpha \in C_{\mathrm{M}}(\alpha)$ and $S C(\alpha) \backslash\{\mathrm{M}\}=S C_{\mathrm{M}}(\alpha) \subseteq \psi_{\mathrm{M}} \alpha$.

\section{Definition 3.1}

$\mathrm{sc}_{\kappa}(\alpha):= \begin{cases}\max S C_{\mathrm{M}}(\alpha) & \text { if } \kappa=\mathrm{M} \& S C_{\mathrm{M}}(\alpha) \neq \emptyset \\ 0 & \text { otherwise }\end{cases}$

\section{Lemma 3.1}

a) $\operatorname{sc}_{\kappa}(\alpha)<\psi_{\kappa} \alpha$

b) $\pi=\mathrm{M} \& \mathrm{sc}_{\pi}(\beta)<\psi_{\kappa} \alpha \Longrightarrow \beta \in C_{\kappa}(\alpha)$

Proof. Trivial (cf. L.1.a,e,f and L.1.4a).

\section{Lemma 3.2}

Let $\kappa \in C_{\kappa}(\alpha) \& \pi \in C_{\pi}(\beta)$. Then

$\psi_{\pi} \beta<\kappa<\pi \& \mathrm{sc}_{\pi}(\beta)<\psi_{\kappa} \alpha \Longrightarrow \psi_{\pi} \beta<\psi_{\kappa} \alpha$

Proof. By L.1.4c,d it follows that $\Omega_{\pi}=\pi$ and $\Omega_{\psi_{\pi} \beta}=\psi_{\pi} \beta$. Therefore if $\kappa=\Omega_{\sigma+1}$ then $\psi_{\pi} \beta \leq \Omega_{\sigma}<\psi_{\kappa} \alpha$, and we may now assume that $\Omega_{\kappa}=\kappa$. Then by L.1.2 and L.1.3b we obtain $\kappa=\psi_{\mathrm{M}} \gamma$ with $\gamma<\alpha \& \gamma \in C_{\kappa}(\alpha) \cap C_{\mathrm{M}}(\gamma)$. By L.1.4a and L1.3a we get $S C_{\mathrm{M}}(\gamma) \subseteq C_{\kappa}(\alpha) \cap C_{\mathrm{M}}(\gamma) \cap \mathrm{M}=C_{\kappa}(\alpha) \cap \kappa=\psi_{\kappa} \alpha$. From $\psi_{\pi} \beta<\kappa=\psi_{\mathrm{M}} \gamma<\pi$ it follows that $\psi_{\mathrm{M}} \gamma \notin C_{\pi}(\beta)$ and thus $\beta \leq \gamma$ or $\psi_{\pi} \beta \leq \mathrm{sc}_{\mathrm{M}}(\gamma)$. - If $\psi_{\pi} \beta \leq \mathrm{sc}_{\mathrm{M}}(\gamma)$ then $\psi_{\pi} \beta<\psi_{\kappa} \alpha$, since $S C_{\mathrm{M}}(\gamma) \subseteq \psi_{\kappa} \alpha$. If $\operatorname{sc}_{\mathrm{M}}(\gamma)<\psi_{\pi} \beta \quad \& \quad \pi=\mathrm{M}$ then we have $\beta \leq \gamma<\alpha$ and $\beta \in C_{\kappa}(\alpha)$ (since $\mathrm{sc}_{\pi}(\beta)<\psi_{\kappa} \alpha$ ), from which we get $\psi_{\pi} \beta \in C_{\kappa}(\alpha) \cap \kappa=\psi_{\kappa} \alpha$. - For $\pi=\mathrm{M}$ the proof is now finished. - If $\mathrm{sc}_{\mathrm{M}}(\gamma)<\psi_{\pi} \beta \quad \& \quad \pi<\mathrm{M}$ then $\psi_{\mathrm{M}} \gamma<\pi<\mathrm{M} \& \mathrm{sc}_{\mathrm{M}}(\gamma)<\psi_{\pi} \beta$ which (according to what we already proved for $\pi=\mathrm{M}$ ) implies $\kappa=\psi_{\mathrm{M}} \gamma<\psi_{\kappa} \alpha$. Contradiction. 


\section{Definition 3.2}

$\mathcal{K}(\pi, \beta, \kappa, \alpha)$ abbreviates the disjunction of $(\mathcal{K} 1), \ldots,(\mathcal{K} 4)$ below:

$(\mathcal{K} 1) \pi \leq \psi_{\kappa} \alpha$

$(\mathcal{K} 2) \quad \psi_{\pi} \beta \leq \mathrm{sc}_{\kappa}(\alpha)$

$(\mathcal{K} 3) \pi=\kappa \& \beta<\alpha \& \mathrm{sc}_{\pi}(\beta)<\psi_{\kappa} \alpha$

$(\mathcal{K} 4) \psi_{\pi} \beta<\kappa<\pi \& \operatorname{sc}_{\pi}(\beta)<\psi_{\kappa} \alpha$

\section{Lemma 3.3}

Let $\kappa \in C_{\kappa}(\alpha) \& \pi \in C_{\pi}(\beta)$.

a) $\neg \mathcal{K}(\pi, \beta, \kappa, \alpha) \& \neg \mathcal{K}(\kappa, \alpha, \pi, \beta) \Longrightarrow \kappa=\pi \& \alpha=\beta$

b) $\mathcal{K}(\pi, \beta, \kappa, \alpha) \Longrightarrow \psi_{\pi} \beta \leq \psi_{\kappa} \alpha$

c) $\mathcal{K}(\pi, \beta, \kappa, \alpha) \& \beta \in C_{\pi}(\beta) \Longrightarrow \psi_{\pi} \beta<\psi_{\kappa} \alpha$

Proof. a) is a logical consequence of the linearity of $<$. b) and c) follow immediately from L.1.3a, L.1.5a, L.1.6, L.3.1, L.3.2.

As an immediate consequence from lemma 3.3 we get

Theorem 3.1

$\kappa, \alpha \in C_{\kappa}(\alpha) \& \pi, \beta \in C_{\pi}(\beta) \& \psi_{\kappa} \alpha=\psi_{\pi} \beta \Longrightarrow \kappa=\pi \& \alpha=\beta$.

\section{Theorem 3.2}

Let $\kappa \in C_{\kappa}(\alpha) \& \pi, \beta \in C_{\pi}(\beta)$.

a) $\psi_{\pi} \beta<\psi_{\kappa} \alpha \Longleftrightarrow \mathcal{K}(\pi, \beta, \kappa, \alpha)$

b) $\psi_{\pi} \beta \in C_{\kappa}(\alpha) \Longleftrightarrow\left(\psi_{\pi} \beta<\psi_{\kappa} \alpha\right.$ or $\left.\left[\beta<\alpha \& \pi, \beta \in C_{\kappa}(\alpha)\right]\right)$

Proof. a) " $\Leftarrow$ " follows from L.3.3b. " $\Rightarrow$ " follows from L.3.3a,c.

b) The " $\Leftarrow$ " part is trivial. So let us assume that $\psi_{\kappa} \alpha \leq \psi_{\pi} \beta \in C_{\kappa}(\alpha)$. By

L.1.2 and L.1.3c this implies the existence of $\tau, \xi \in C_{\kappa}(\alpha) \cap C_{\tau}(\xi)$ with $\xi<\alpha$ and $\psi_{\pi} \beta=\psi_{\tau} \xi$. From this by Theorem 3.1 we obtain $\pi=\tau \in C_{\kappa}(\alpha)$ and $\beta=\xi \in C_{\kappa}(\alpha) \cap \alpha$.

\section{Theorem 3.3}

$\kappa \in C_{\kappa}(\alpha) \Longleftrightarrow \kappa \in\left\{\Omega_{\sigma+1}: \sigma<\mathrm{M}\right\} \cup\left\{\psi_{\mathrm{M}} \xi: \xi<\alpha\right\} \cup\{\mathrm{M}\}$

Proof. 1. " $\Rightarrow$ " follows from L.1.2 and L.1.3b. - 2. By L.1.3d we have $\left(\kappa \in C_{\kappa}(\alpha) \Leftrightarrow \kappa \in C(\alpha, \kappa)\right)$. - 3. If $\kappa=\Omega_{\sigma+1}$ then $\sigma+1<\kappa$ and thus $\kappa \in C(\alpha, \kappa)$. - 4. If $\kappa=\psi_{\mathrm{M}} \xi$ with $\xi<\alpha$ then $\xi \in C_{\mathrm{M}}(\xi)=C(\xi, \kappa) \subseteq C(\alpha, \kappa)$ and thus $\kappa \in C(\alpha, \kappa)$. 


\section{Theorem 3.4}

$\kappa=\Omega_{\sigma+1} \Longrightarrow C_{\kappa}(\alpha)=C\left(\alpha, \Omega_{\sigma}+1\right)$

Proof by induction on $\alpha$. So let us assume that $C_{\kappa}(\xi)=C\left(\xi, \Omega_{\sigma}+1\right)$, for all $\xi<\alpha$. - We have to prove $\psi_{\kappa} \alpha \subseteq C\left(\alpha, \Omega_{\sigma}+1\right)$. As we will show below the I.H. implies that $\beta:=C\left(\alpha, \Omega_{\sigma}+1\right) \cap \kappa$ is in fact an ordinal. Obviously $\kappa \in C(\alpha, \beta)$ and $C(\alpha, \beta) \cap \kappa \subseteq C\left(\alpha, \Omega_{\sigma}+1\right) \cap \kappa=\beta$ and thus $\psi_{\kappa} \alpha \leq \beta$, i.e. $\psi_{\kappa} \alpha \subseteq C\left(\alpha, \Omega_{\sigma}+1\right)$. - CLAIM: $\gamma \in C\left(\alpha, \Omega_{\sigma}+1\right) \cap \kappa \Longrightarrow \gamma \subseteq C\left(\alpha, \Omega_{\sigma}+1\right)$. Proof. 1. $\Omega_{\sigma}<\gamma \in$ SC. Then $\gamma=\psi_{\pi} \xi$ with $\xi<\alpha \quad \& \quad \xi \in C_{\pi}(\xi)$. Since $\Omega_{\sigma}<\gamma<\kappa=\Omega_{\sigma+1}$, we have $\pi=\kappa$ and therefore by the above I.H. $C_{\kappa}(\xi)=C\left(\xi, \Omega_{\sigma}+1\right)$. Hence $\gamma=\psi_{\kappa} \xi \subseteq C\left(\xi, \Omega_{\sigma}+1\right) \subseteq C\left(\alpha, \Omega_{\sigma}+1\right)$.

2. Let $\gamma$ be arbitrary and $\gamma_{0}:=\max (\{0\} \cup S C(\gamma))$. Then (by 1. above) $\gamma_{0} \cup\left\{\gamma_{0}\right\} \subseteq C\left(\alpha, \Omega_{\sigma}+1\right)$. From this we get $\gamma \subseteq \gamma^{*} \subseteq C\left(\alpha, \Omega_{\sigma}+1\right)$, where $\gamma^{*}:=\min \left\{\eta \in \mathrm{SC}: \gamma_{0}<\eta\right\}$.

COROLLARY. $\psi_{\Omega_{1}} \alpha=C(\alpha, 0) \cap \Omega_{1}$

\section{References}

[1] Buchholz,W.: A new system of proof-theoretic ordinal functions. Annals of Pure and Applied Logic 32 (1986), 195-207.

[2] Buchholz,W.: A simplified version of local predicativity. In: Proceedings Proof Theory Meeting, Leeds '90. To appear.

[3] Buchholz,W. and Schütte, K.: Ein Ordinalzahlsystem für die beweistheoretische Abgrenzung der $\Pi_{2}^{1}$-Separation und Bar-Induktion. Sitzungsberichte der Bayerischen Akademie der Wissenschaften, MathematischNaturwissenschaftliche Klasse (1983)

[4] Jäger,G.: $\rho$-inaccessible ordinals, collapsing functions and a recursive notation system. Arch. math. Logik 24 (1984), 49-62.

[5] Rathjen,M.: Ordinal Notations Based on a Weakly Mahlo cardinal. Arch. math. Logic 29 (1990), 249-263.

[6] Rathjen,M.: Proof-Theoretic Analysis of KPM. Arch. math. Logic 30 (1991), 377-403. 
Mathematisches Institut der Universität München

Theresienstr. 39, D-8000 München 2, Germany 